\title{
Interactive Example-Based Terrain Authoring with Conditional Generative Adversarial Networks
}

\author{
ÉRIC GUÉRIN, Univ Lyon, INSA-Lyon, CNRS, LIRIS \\ JULIE DIGNE, Univ Lyon, CNRS, LIRIS \\ ÉRIC GALIN, Univ Lyon, Université Lyon 1, CNRS, LIRIS \\ ADRIEN PEYTAVIE, Univ Lyon, Université Lyon 1, CNRS, LIRIS \\ CHRISTIAN WOLF, Univ Lyon, INSA-Lyon, CNRS, LIRIS \\ BEDRICH BENES, Purdue University \\ BENOÎT MARTINEZ, Ubisoft Entertainment
}

\begin{abstract}
Authoring virtual terrains presents a challenge and there is a strong need for authoring tools able to create realistic terrains with simple user-inputs and with high user control. We propose an example-based authoring pipeline that uses a set of terrain synthesizers dedicated to specific tasks. Each terrain synthesizer is a Conditional Generative Adversarial Network trained by using real-world terrains and their sketched counterparts. The training sets are built automatically with a view that the terrain synthesizers learn the generation from features that are easy to sketch. During the authoring process, the artist first creates a rough sketch of the main terrain features, such as rivers, valleys and ridges, and the algorithm automatically synthesizes a terrain corresponding to the sketch using the learned features of the training samples. Moreover, an erosion synthesizer can also generate terrain evolution by erosion at a very low computational cost. Our framework allows for an easy terrain authoring and provides a high level of realism for a minimum sketch cost. We show various examples of terrain synthesis created by experienced as well as inexperienced users who are able to design a vast variety of complex terrains in a very short time.
\end{abstract}

CCS Concepts: • Computing methodologies $\rightarrow$ Shape modeling;

Additional Key Words and Phrases: Procedural modeling, Terrain generation, Deep Learning

ACM Reference format:

Éric Guérin, Julie Digne, Éric Galin, Adrien Peytavie, Christian Wolf, Bedrich Benes, and Benoît Martinez. 2017. Interactive Example-Based Terrain Authoring with Conditional Generative Adversarial Networks. ACM Trans. Graph. 36, 6, Article 228 (November 2017), 13 pages.

https://doi.org/10.1145/3130800.3130804

\section{INTRODUCTION}

Despite more than thirty years of research in terrain modeling, authoring virtual terrains by using contemporary techniques remains a demanding task. One reason for this difficulty is the wide variety of geomorphological processes that control the terrain shape formation. Terrains are exposed for thousands of years to different erosion and land-forming agents such as water erosion, varying temperatures, vegetation, that are difficult to express by simple and versatile algorithms that could be used as editing tools. This brings

This work is supported by the project PAPAYA P110720-2659260, funded by the Fonds National pour la Societé Numérique, and the project HWD ANR-16-CE33-0001, supported by Agence Nationale de la Recherche. We thank Howard Zhou for allowing us to use the DEM and sketch of Fig. 18.

(c) 2017 Association for Computing Machinery.

This is the author's version of the work. It is posted here for your personal use. Not for redistribution. The definitive Version of Record was published in ACM Transactions on Graphics, https://doi.org/10.1145/3130800.3130804. many important challenges, among them expressing the designer's intent and modeling large-scale detailed terrains while allowing tight user-control. Generating a new terrain is a complex and demanding process and it is even more complicated to do it intuitively and quickly.
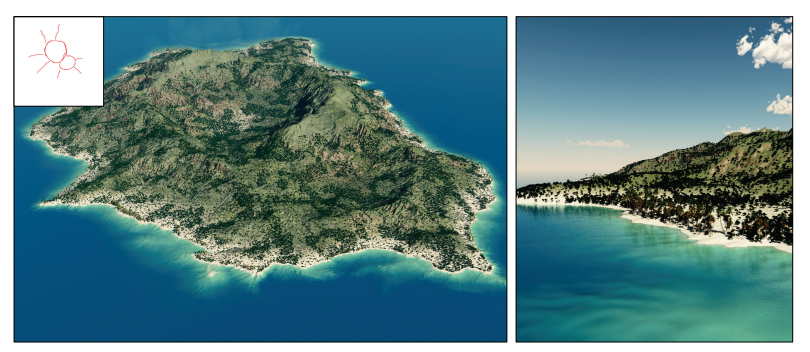

Fig. 1. A volcanic terrain generated by our method created by an inexperienced user from a few strokes depicting the crest lines.

A tremendous progress has been achieved in terrain modeling and the existing techniques can be categorized into procedural, simulation-based, sketch-based, and example-based. Many terrains have strong fractal features and while procedural methods allow for fast terrain generation, they often fail to provide control over the terrain features. While simulation-based methods (such as erosion and hydrology-based algorithms) generate geologically correct models, they often lack user control and are computationally expensive. Sketch-based methods provide a high level of control, but they do not generate geologically correct outputs and editing is tedious for large terrains. Existing example-based methods can generate large terrains using small examples but provide low user-control. Moreover, they replicate the exemplars, but do not easily generate new features. One important common drawback of the existing algorithms is that they cannot be easily applied to large-scale terrains.

Recent progress in Deep Learning provided solutions to many hard problems in Computer Graphics and Vision, not only for classification but also for synthesis. One of the most powerful generative methods is the so-called Generative Adversarial Network (GAN) [Goodfellow et al. 2014] and one of its extensions, conditional GAN (cGAN).

We integrate cGANs in a fast example-based approach to realistic terrain synthesis (Fig. 1) that is oblivious to the underlying 

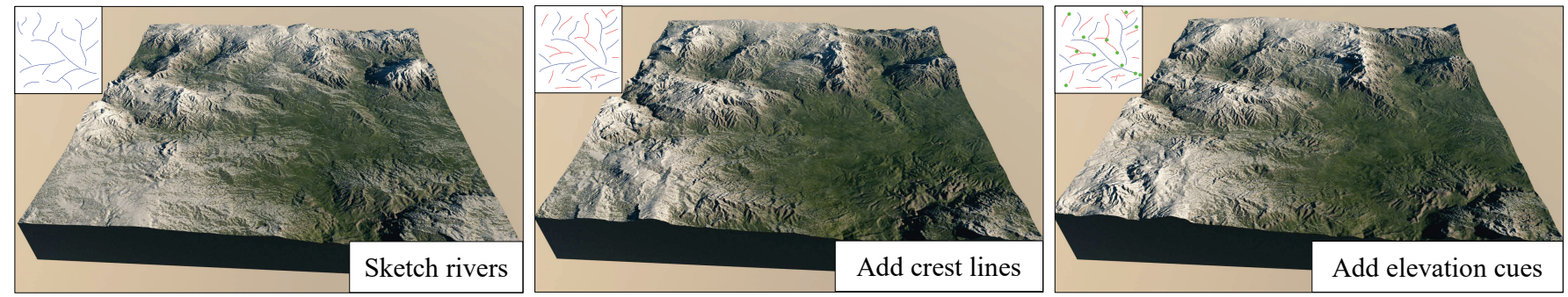

Fig. 2. Our interactive terrain modeling framework allows the user to quickly, easily, and intuitively author realistic terrain models by using sketches of crest lines, rivers, or iso contours. Our method consists of a training step and an interactive sketching step. During the training step, we analyze a large number of terrains and extract geometric features that will serve as sketching elements. The sketches are fed to a set of cGANs which learn various generative processes. During the authoring step, the pre-trained networks are used to synthesize a terrain model that matches the input sketch and the incremental edits.

geological phenomena. Our method bridges the gap between the intuitiveness and flexibility of interactive authoring processes, while providing efficiency and expressive power. We do not attempt to model the underlying geomorphologic phenomena, but instead we use construction by example.

The cGAN training requires large training sets and it would not be feasible to ask artists to provide hundreds of terrain sketches. Instead, we propose to extract automatically sketches from realworld terrains. These sketches combine visually important features such as crests, valleys and river networks that have clear user intuition and can be easily sketched. We then train several cGANs with examples built from pairs of real terrains and their automatically generated sketches. Each cGAN yields what we call a terrain synthesizer capable of generating the entire elevation of a terrain from input data.

Terrains are also shaped by erosion, a geomorphological process which is notoriously slow. Although GPU implementations of erosion algorithm exist, they cannot be efficiently applied to large terrains. In our work we propose an erosion meta-simulation (simulation of a simulation) by training a dedicated cGAN. This allows to apply erosion to large terrains time-efficiently.

In our approach, the user starts the interactive editing session by providing a rough sketch of a terrain and the terrain synthesizer generates the corresponding large-scale terrain model. The result can be later modified by editing the sketch, erasing and regenerating terrain parts, by our eraser synthesizer. A key feature of the entire process is its efficiency: during the authoring session, every terrain generation takes only a few milliseconds which allows interactive feedback to the designer. An example in Fig. 2 shows the usage of our framework: starting with a river network, adding crests and elevation cues generates a real-time terrain in each step.

The main contributions of our work are threefold: 1) we propose the first terrain authoring pipeline driven by real world examples based on Deep Learning, 2) we present an automatic sketches generation process to build learning datasets, and finally, 3) we show that our approach can be used to learn complex natural processes, such as hydraulic erosion. We evaluated our approach by providing a large variation of landscape types (alpine mountain, volcanic island, canyon) and we also conducted a qualitative user study that confirms the ease of use of our technique.

\section{RELATED WORK}

Terrain modeling has been a focus of computer graphics for a long time and the existing body of work can be classified into procedural, simulation-based, and example-based techniques. We refer the reader to [Natali et al. 2013] for an overview of terrain modeling representations and to [Smelik et al. 2014] for a survey of procedural terrain modeling. We also briefly review Machine Learning algorithms and especially Deep Learning algorithms.

Classical procedural methods are computationally efficient, because they usually use some kind of fractal noise that visually resembles real terrains. Fractals are useful for modeling terrains that are self-similar and can be observed as fresh and not eroded [Fournier et al. 1982]. Fractal interpolation was used to complete user-defined river networks [Kelley et al. 1988] and models generated by Lsystems [Prusinkiewicz and Hammel 1993]. They were later used to generate fractal terrains constrained by user-prescribed rivers trajectories [Belhadj and Audibert 2005] and planets featuring procedurally generated river networks [Derzapf et al. 2011]. A major limitation of the procedural models is their control. Fractals are defined by seeding a random number generator and the result is difficult to predict. User control of fractal methods has been addressed by several works. In particular by defining terrains from feature curves such as river networks and ridges [Génevaux et al. 2013; Hnaidi et al. 2010]. Recently, Génevaux et al. [2015] introduced a hierarchical distribution tree that models the terrain as a distribution of primitives that are procedurally blended, carved, and warped together. Moreover, it was quickly noticed that real terrains do not always conform to pure fractal description because they are exposed to various morphogenesis phenomena, among them erosion plays the most important role.

Simulation-based methods were introduced by the work of Musgrave et al. [1989] who used various kinds of erosion to modify fractal terrains. Erosion algorithms were later extended by hydraulic erosion in [Chiba et al. 1998; Nagashima 1998]. Height fields [Cordonnier et al. 2016] are the most common representations used for erosion simulation, although layered data structures [Benes and Forsbach 2001], volumetric data [Benes et al. 2006], and smoothed particle hydrodynamics [Krištof et al. 2009] were also used. Smallscale volumetric erosion was also used to simulate cliffs [Peytavie et al. 2009]. Recently, Cordonnier et al. [2017a] used simulations in 

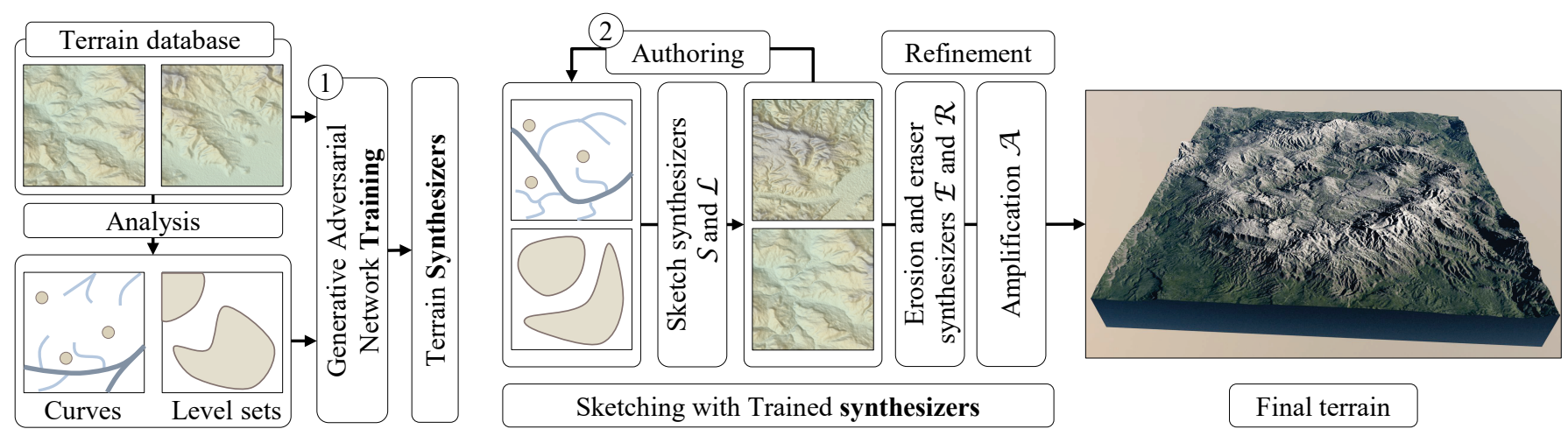

Fig. 3. Our system is a two stage process. During the training step, we analyze a large input set of example terrains and extract features such as ridge lines, river networks, levelsets or points of interest. The data and the input terrains are used to train a set of conditional Generative Adversarial Networks that learn the correlations between the terrains and the sparse data. During the interactive authoring session, the network synthesizes the terrain from the user sketch.

interactive setting to generate large-scale terrains affected by subsurface tectonics. Erosion algorithms were brought at interactive frame rate by using GPUs in [Mei et al. 2007; Vanek et al. 2011] While simulation-based algorithms generate geologically correct models, they are difficult to control and they are computationally demanding. Moreover, it is difficult to express a user intent by using those methods.

Example-based and user-defined approaches use existing terrains or user sketches to define terrain models. A typical representative of the example-based methods is the approach of [Zhou et al. 2007] that uses the height field patches from a real terrain and combines them into a user-defined sketch. Various methods use high-level interactive inputs to define terrains. Silhouettes were used to define roughness [Gain et al. 2009, 2015] or to deform an existing terrain to create a view from a certain viewpoint [Tasse et al. 2014]. Sketch-based approaches [Gain et al. 2009; Hnaidi et al. 2010; Tasse et al. 2012] or direct interactive terrain editing [Peytavie et al. 2009] were used to define terrains with a high level of control, but can generate terrains that are not geologically correct. Hybrid approaches combine interactive editing with simulations [Vanek et al. 2011; Štava et al 2008] but they are limited to small scenes. Sketch-based methods involve manual editing that can be tedious whereas example-based algorithms are limited by the input exemplars.

Machine learning has been used to generate images and other high dimensional and structured data similar to a training set for texture synthesis [Gatys et al. 2015; Kwatra et al. 2003], to generate images of a given type [Gregor et al. 2015], to predict future video frames [Mathieu et al. 2016], or to use sketches to complete procedural buildings [Nishida et al. 2016]. Earlier work was based on dictionary learning [Rubinstein et al. 2010] or graphical models like $\mathrm{MRF}$ /CRFs. These models are limited by the required optimization stage during decoding, resulting in scalar hidden states and low order interaction between output variables [Kwatra et al. 2003].

Neural networks overcome this limitation by dealing with structured data differently. In the most widely used formulations, no optimization is required during decoding, which allows the model to (i) resort to a rich componential hidden state and to (ii) use complex interactions between output variables and hidden states. Methods have been proposed which learn a direct (often convolutional) mapping between input and output, e.g. [Dosovitskiy et al. 2015], to generate images of 3D models given object type, viewpoint and color. Probabilistic graphical models (and neural networks), such as Variational Auto-Encoders (VAE), put a strong emphasis on modeling stochastic latent space [Kingma and Welling 2014]. Because they are capable of modeling highly complex interactions, they can also be used to generate images [Gregor et al. 2015; Mansimov et al. 2016]. U-nets [Huang et al. 2016; Ronneberger et al. 2015] map input to output by first decreasing spatial resolution iteratively through a bottleneck and then restoring spatial resolution with upsampling, Unets benefit from additional skip-connections between layers with the same resolution.

Ulyanov [2016] used generative convolutional networks to provide textures in multiple resolutions. Li and Wand [2016] used GANs to generate textures without blending, and [Zhu et al. 2016] showed how to use GANs for texturing meaningful manifolds.

The recently proposed Generative Adversarial Networks (GANs) [Goodfellow et al. 2014] are also of stochastic nature. They train two competing networks, a generator network $\mathcal{G}$, able to generate new examples, and a discriminator $\mathcal{D}$, able to discriminate between real examples and generated examples. The generator learns to create realistic looking images which the discriminator is unable to distinguish from the images of the training set. Although VAE provides additional control over the latent space which might help to better enforce constraints on the output, GANs currently produce data of higher quality, which is due to their adversarial loss. Conditional GANs can process additional inputs which allows to learn relationships between pairs of images [Isola et al. 2016]. This has been successfully applied to digital image generation and completion [Mirza and Osindero 2014; Pathak et al. 2016]. Our method also makes use of a conditional GAN to train various terrain synthesizers from carefully designed input samples built from real-world examples. To the best of our knowledge, deep neural networks were not used for terrain synthesis before. 


\section{OVERVIEW}

Our method consists of a training pre-processing step and an interactive authoring step (Fig. 3). The pre-processing step uses a set of example data-sets to produce a set of units called Terrain Synthesizers that are at the heart of our pipeline. A Terrain Synthesizer takes an input sketch or annotated terrain and produces an output or modified terrain (Section 4).

We introduce four different terrain synthesizers. The sketch-toterrain synthesizer $\mathcal{S}$ creates a terrain from a sketch containing ridges, rivers, altitude cues or any combination of the three; the levelset-to-terrain synthesizer $\mathcal{L}$ turns a binary levelset image into a terrain; the eraser synthesizer $\mathcal{R}$ removes a user-specified part of the terrain and completes it, and, finally, the erosion synthesizer $\mathcal{E}$ transforms an input terrain into the corresponding eroded terrain. Because each synthesizer is specialized in a specific task, we need to build a set of dedicated databases from real-world examples to learn each synthesis (Section 5). The training step, performed once and for all, is particularly important since the quality and realism of the terrain produced in an authoring session is strongly correlated with the learned synthesis ability. Interestingly, one could easily add Terrain Synthesizers to the pipeline: for example to consider other kinds of sketches.

The authoring stage (Section 6) starts by loading the terrain synthesizers that are used during the authoring session. The input to our framework is a coarse sketch and the output is a 3D model of a terrain that is created by successive user-edits and optionally adding erosion to the final result. An artist will start by providing a coarse sketch features such as rivers, ridges, some altitude cues, or a combination of them. The input is given to the sketch-to-terrain synthesizer that generates a plausible terrain from it. If the result is not satisfactory, the user can re-edit the sketch and rerun the synthesis or remove parts of the terrain that will then be completed by the eraser synthesizer. After the coarse sketch is finished, the user can erode the terrain by running the erosion synthesizer.

Once the user is satisfied with the generated large-scale terrain, small scale details can be added by using a super-resolution technique. We have used an algorithm from [Guérin et al. 2016] to add small-scale details to an existing terrain. We will refer to this method as terrain amplification as it considers the information in the terrain in order to amplify it. This method is particularly well suited for the terrains generated by cGANs, because they contain coherent landforms features, but lack small scale details.

\section{TERRAIN SYNTHESIZER}

A key feature of our approach lies in its ability to synthesize terrains from various types of inputs. In particular, we can synthesize a terrain from different kinds of sketches, complete a terrain with missing data or parts erased during interactive editing, or synthesize an eroded terrain from another input terrain. All these problems can be interpreted as learning a way to predict an output B from an input A. In our setting all terrains are represented as Digital Elevation Models (DEMs) and sketches are represented as images.

Our approach builds on Conditional Generative Adversarial Networks (cGAN) [Isola et al. 2016]. cGANs are pairs of deep networks, a generative network $\mathcal{G}$ able to generate $\mathrm{B}$ from $\mathrm{A}$ and a discriminative network $\mathcal{D}$ able to discriminate between real pairs (A, B), i.e., data from the training set, and fake ones $(\mathrm{A}, \mathcal{G}(\mathrm{A}))$, data generated by the network $\mathcal{G}$. The name adversarial training derives from the fact that $\mathcal{G}$ is trained to fool $\mathcal{D}$ and that $\mathcal{D}$ tries to avoid being fooled by $\mathcal{G}$. While we are mostly interested in the generative network $\mathcal{G}$,

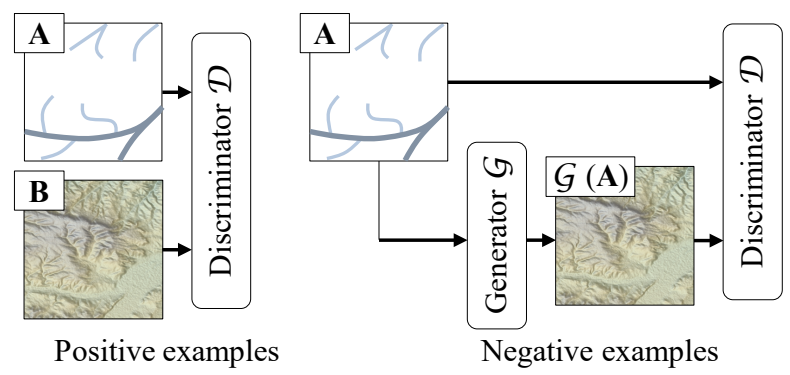

Fig. 4. Overview of the training of a cGAN: The discriminator $\mathcal{D}$ learns to classify between real and synthesized pairs, whereas the generator learns to fool the discriminator.

$\mathcal{D}$ is crucial to the learning stage because its discriminative power conditions the quality of the generator $\mathcal{G}$. Indeed, the generative network can only become efficient in producing real examples if $\mathcal{D}$ is efficient at discriminating real and fake examples. Failure to do so leads to a high error on $\mathcal{D}$ 's side for wrong reasons: the discriminators own abilities vs. the quality of the generator's output. Thus, while $\mathcal{G}$ maps images to DEMs, $\mathcal{D}$ operates patchwise and classifies the patches (of size $70 \times 70$ ) of the test pair as either real $(=1)$ or fake $(=0)$ and outputs the average over the binary decisions for all patches. If $\mathbf{B}$ is a real-world DEM and $\mathbf{A}$ its extracted sketch, the estimated classification $\mathcal{D}(\mathrm{A}, \mathrm{B})$ should be close to 1 . Conversely, the discriminator should learn to recognize a synthesized pair $(\mathrm{A}, \mathcal{G}(\mathrm{A}))$ and tend to zero in that case. Following [Isola et al. 2016], this is captured by the following objective function:

$$
\mathbb{E}_{(\mathrm{A}, \mathrm{B})}[\log \mathcal{D}(\mathrm{A}, \mathrm{B})]+\mathbb{E}_{\mathrm{A}}[\log (1-\mathcal{D}(\mathrm{A}, \mathcal{G}(\mathrm{A})))] .
$$

The expectation is taken over the distribution of the data (A, B). Equation (1) is maximized over the discriminator parameters. The generator should in turn learn to generate DEMs which minimize the discriminator objective (for the discriminator's best effort), which turns the learning process for the generator parameters into a minimax game:

$$
\min _{\mathcal{G}} \max _{\mathcal{D}} \mathbb{E}_{(\mathrm{A}, \mathrm{B})}[\log \mathcal{D}(\mathrm{A}, \mathrm{B})]+\mathbb{E}_{\mathrm{A}}[\log (1-\mathcal{D}(\mathrm{A}, \mathcal{G}(\mathbf{A})))] .
$$

The above adversarial objective with respect to $\mathcal{G}$ does not use the ground truth image $\mathrm{B}$, but checks (through the discriminator) whether the generated image lies on the data manifold. In practice, this does not provide enough supervision for efficient learning. To alleviate this lack of supervision, it is common to add a regularization term involving the ground truth image $\mathbf{B}$ to the objective, e.g. gradient difference loss [Mathieu et al. 2016] or $L_{1}$ regularization $\|\mathbf{B}-\mathcal{G}(\mathbf{A})\|_{1}$, as in [Isola et al. 2016]. We chose the latter formulation. The additional term only has an impact on the generator training. 

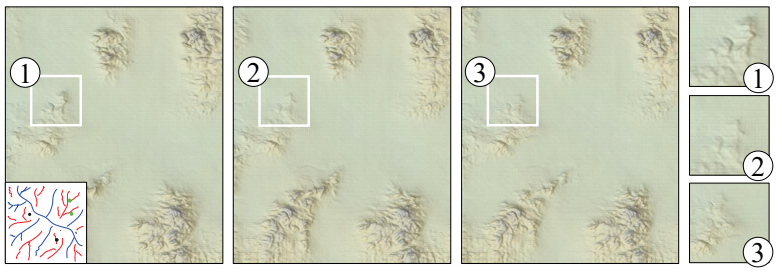

Fig. 5. The same input produces slightly different outputs when executed several times. While small scale details can be different, we observed that the main landforms features remain the same.

Contrary to standard GAN formulations, we do not add any noise input to the generator $\mathcal{G}$, and provide noise (for stochasticity) only in the form of dropout [Krizhevsky et al. 2012], i.e. switching off units randomly during training and testing with probability $p=0.5$ Thus running the synthesizer twice will yield slightly different results. In our experiments, we observed that the results only slightly varied when running the synthesizer on the same input several times (Fig. 5).

The network architectures have been adapted from [Radford et al. 2016] and [Isola et al. 2016]. The generator architecture is an encoder-decoder network with an encoding part composed of a sequence of fully-convolutional layers (convolutions filters of size $4 \times 4$ ) and resolution reductions, and a decoder composed of a sequence of deconvolutions/upsampling. In the decoding part, each layer is thus connected to a layer of lower resolution, and additional skip-connections connect it to the encoder layer of identical resolution (U-net). These additional connections allow to bypass the encoder-decoder bottleneck by transmitting low-level information from the input directly to the output. The number of features in the generator in the successive layers is: $64,128,256,512,512,512$, and 512. There is a single output channel storing the terrain altitude, and the number of input channels varies from one (for levelset and erosion synthesizers) to three (for sketch and eraser synthesizers).

Our training follows the Image-to-Image network training [Isola et al. 2016]. We use a stochastic gradient descent with mini-batches of size one, the Adam optimizer [Kingma and Ba 2015], and the batch normalization [Ioffe and Szegedy 2015]. We alternate between gradient updates of the generator and gradient updates of the discriminator [Goodfellow et al. 2014]. We use up to 500 epochs, which produces less artifacts in the output terrain. In order to introduce more variability to the training data, we randomly use both vertical and horizontal flipping in addition to the standard cropping area.

\section{TRAINING}

Our framework needs to learn mappings from sketches to real world terrains from a set of training pairs $\{(\mathrm{A}, \mathrm{B})\}$ that are input to both networks (Fig. 4). Since we cannot ask an artist to generate a large database of sketch-terrain pairs, we use an automatic generation of those pairs which will govern the quality of the output terrain.

Our approach takes a real-world terrain as B, uses a dedicated algorithm that generates $\mathrm{A}$, and adds the pair $(\mathrm{A}, \mathrm{B})$ to the training set. Notice that the trained synthesizers scales are bound by the scale of the training terrain datasets. In this section, we describe our automatic training set generation. The data source and statistics about the training process are detailed in Section 7.1.

\subsection{Sketch-to-terrain Synthesizer Training}

Sketches can contain altitude cues, rivers, mountain ridges, or any combination of these.

River networks are obtained by simulating the water flow on a terrain and detecting the pixels with high water accumulation. We generate river networks from the terrain elevation using a modified river channel network algorithm inspired by [Tarboton et al. 1991]. We seed water over all the grid points of the terrain and simulate flow using a modified steepest descent D8 algorithm, which routes all flow to the neighboring point to which there is the steepest downward slope [O'Callaghan and Mark 1984].

In order to prevent the water from following always the same path, we use a stochastic direction at every step where the probability is proportional to the height difference between the current pixel and the candidate neighbor. The local minima are processed stochastically: water stuck in a local minimum leaves it with a low probability, or simply disappears. This process yields very precise river networks which may be problematic because it does not correspond to a real user sketch of a river network. A user would indeed provide coarse directions to the river network and would not draw every single river twist.

To alleviate this effect, the terrain is blurred and down-sampled before the flow simulation and the resulting water accumulation is up-sampled to get the rivers at the initial resolution. A final morphological operation is applied to the result in order to obtain a clean 1-pixel width skeleton. Hence the synthesizer training algorithm is provided with inputs that are coarse river directions and it learns to not strictly respect constraints, thus allowing more flexibility in the generator. Fig. 6 shows examples of terrains and their detected river network.

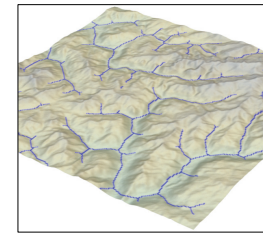

Rivers

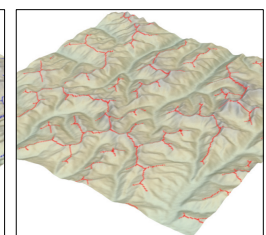

Crest lines

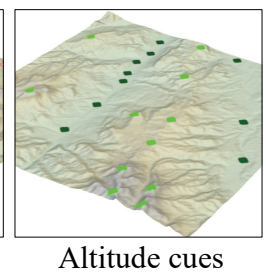

Altitude cues
Fig. 6. Extracted features from different input terrains.

This pre-processing has a great impact on the training, as illustrated in Fig. 8 which shows examples of generated terrains when the dataset has not been blurred prior to the feature extraction. In this case, the extracted features that feed the training are more precise and dense. In this over-constrained context, the synthesizer fails at reconstructing a terrain when the sketch in not dense enough, and even with a greater number of strokes, the generated terrain does not follow the sketch. Using blurred terrains for feature extraction thus makes the synthesis more robust to imprecise and sparser sketches. 

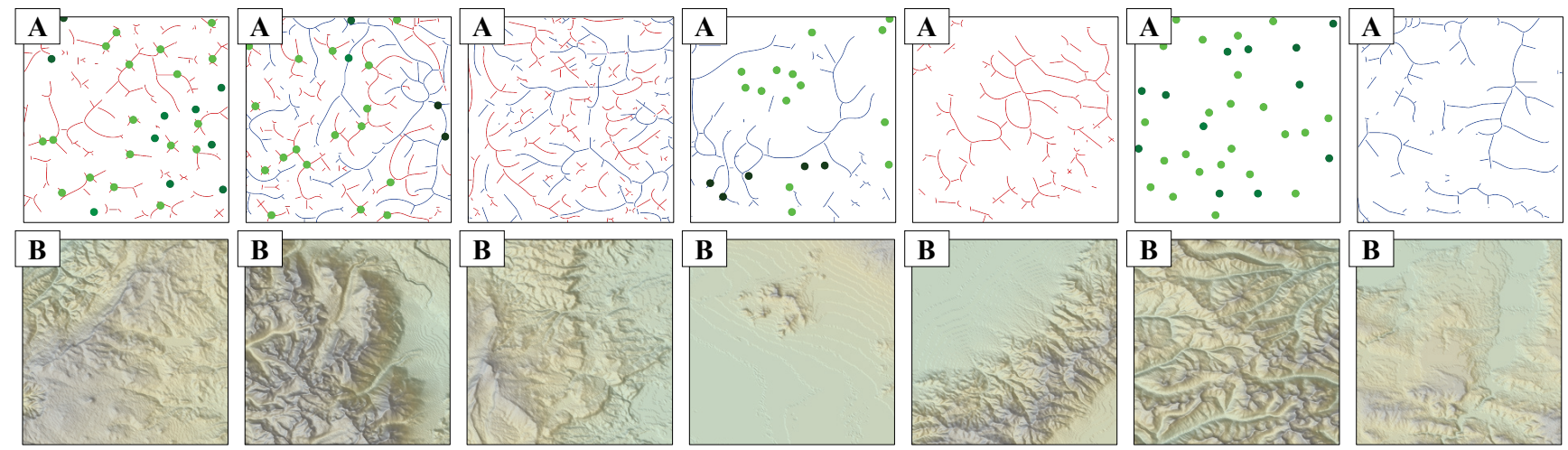

Fig. 7. Different kinds of multichannel sketches generated for the sketch-to-terrain training set.

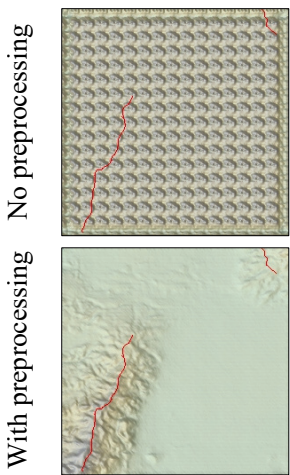

2 strokes

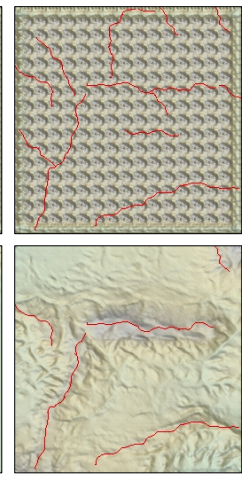

5 strokes
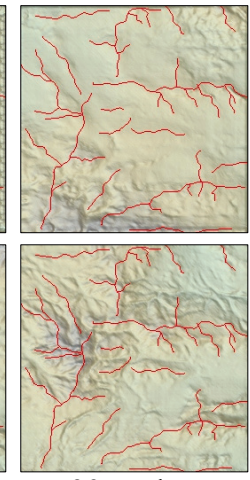

39 strokes
Fig. 8. Comparison of synthesis results when the network is trained with features extracted on non-blurred data (top) and blurred data (bottom). In the first case, the synthesizer needs more input sketches to reconstruct the terrain (top right). With a small number of strokes, visual artifacts appear (top left and middle). This can be avoided when blurred terrain data are used to extract the features (bottom row).

Ridges are detected by inverting the terrain and applying the river detection algorithm. It is the opposite operation to river detection and Fig. 6 shows examples of detected ridges.

Altitude cues are computed as a sparse set of peak and basin points over the terrain with an approximate elevation. Basin points are defined as points where the previous water flow accumulated above a chosen threshold. Conversely, peak points can be defined in a similar way by inverting the elevation of the terrain.

The full example set is generated by providing random combinations of the sketch cues by using different color channels of the images. We map the detected river layer to the blue channel, the ridge layer to the red channel, and the altitude to the green channel. If the sketch does not contain one of these cues, the corresponding channel is set to zero. Fig. 7 shows examples of our training pairs.

\subsection{Levelset-to-terrain Synthesizer Training}

As an alternative to sketching ridges, river curves, and altitude cues, the user can provide large areas of constant elevation that we call levelsets. These are provided as binary images that indicate areas in the terrain where the altitude should be above a given percentile of the altitude distribution ( $60 \%$ in our implementation). This levelset synthesizer serves a different purpose and it cannot be used jointly with the sketch-to-terrain synthesizer. Although different percentile choices could be made, we found that $60 \%$ yielded the most intuitive drawing tool. The example set for this training is easily constructed by blurring the DEMs and thresholding the altitude at the provided percentile. Fig. 9 shows three examples of the training pairs. Once again this example generation involves taking a real-world terrain $B$ and creating the corresponding levelset input A as an entry (A, B) to the training stage.
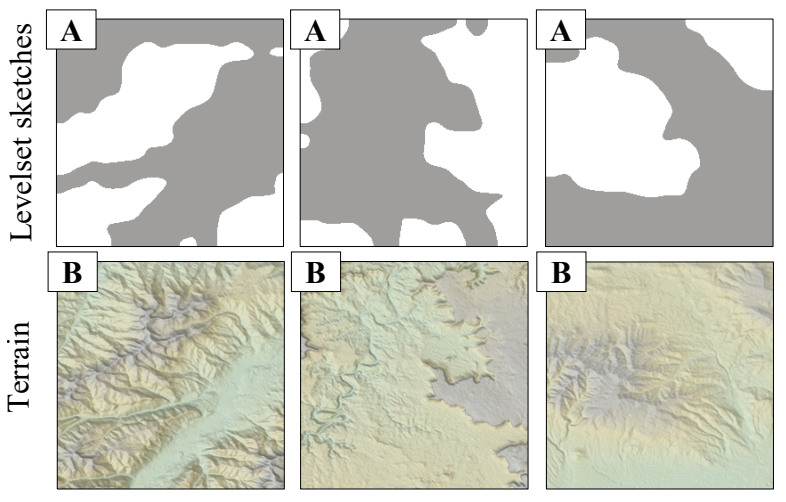

Fig. 9. Levelset examples. The levelset is represented in white.

\subsection{Eraser Synthesizer Training}

Another useful terrain design tool is an eraser synthesizer working directly on the DEM, that removes parts of a terrain and infers its completion. To train this synthesizer, a real-world terrain B is modified through the addition of a random number of disks with 

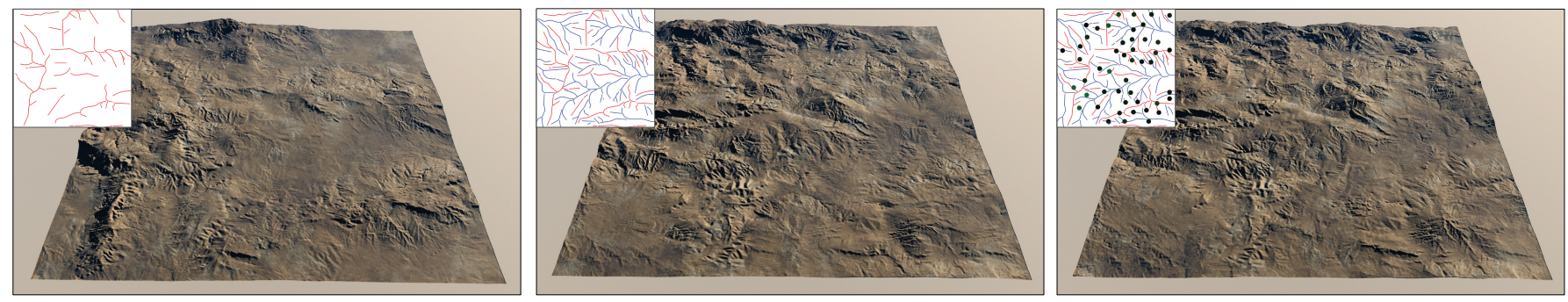

Fig. 10. Example of an interactive authoring session performed by a professional artist: it took him a only a few minutes to design the structure of a large terrain by using ridges (left), adjusting the generated terrain to his intent by incrementally adding rivers (middle), and defining some elevation points (right).

random sizes that define the missing parts. We represent the annotated terrain $\mathrm{A}$ as a two-channel image $\mathrm{Z} \alpha$, where $\mathrm{Z}$ denotes the elevation channel and $\alpha$ the erasure channel. The elevation of the erased terrain part is set to 0 , while the $\alpha$ channel is set to 1 . $\mathrm{B}$ is defined as the complete terrain. The pair $(\mathbf{A}, \mathbf{B})$ is added to the training database for the eraser synthesizer (Fig. 11).

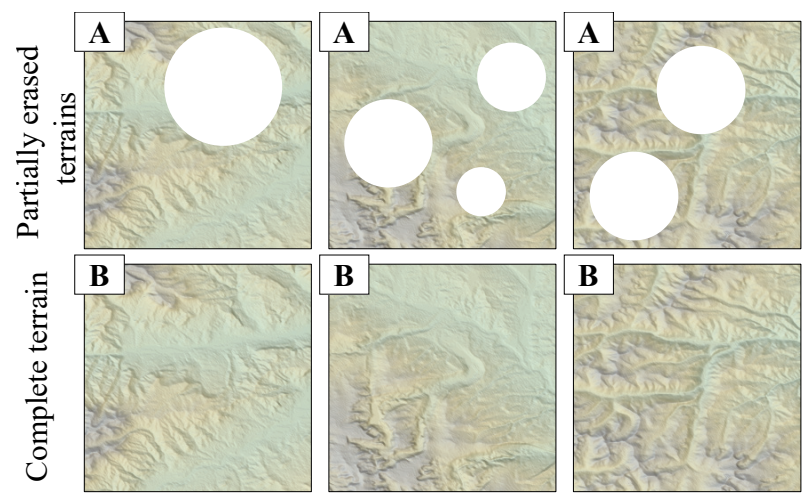

Fig. 11. Eraser synthesizer training example pairs.

\subsection{Erosion Synthesizer Training}

The erosion examples generation proceeds slightly differently from the previous cases. Recall that sketch-to-terrain, levelset-to-terrain, and eraser synthesizers used real-terrains as input B and computed A. However, it is difficult to find real-world data for a terrain and its corresponding eroded version. Instead, we create the erosion examples by taking a real-world terrain as input $\mathbf{A}$ and computing the corresponding data $\mathbf{B}=e(\mathbf{A})$ by simulating erosion $e$ over $\mathbf{A}$ (Fig. 12).

Our approach consists in learning an algorithm that mimics the behavior of a simulation. Inspired by [Cordonnier et al. 2017b], we simulate both interleaved large-scale hydraulic and thermal erosion. Our terrain-erosion model relies on a discrete layered model representing different materials (bedrock, rocks and fine grain sediments). Temperature variations and rainfall trigger aging and weathering events, such as water runoff transporting sediments, or fracture of the bedrock into rock-slides. The simulation computes the evolution of the layered model by stochastically applying a large number of events to the cells of the terrain.
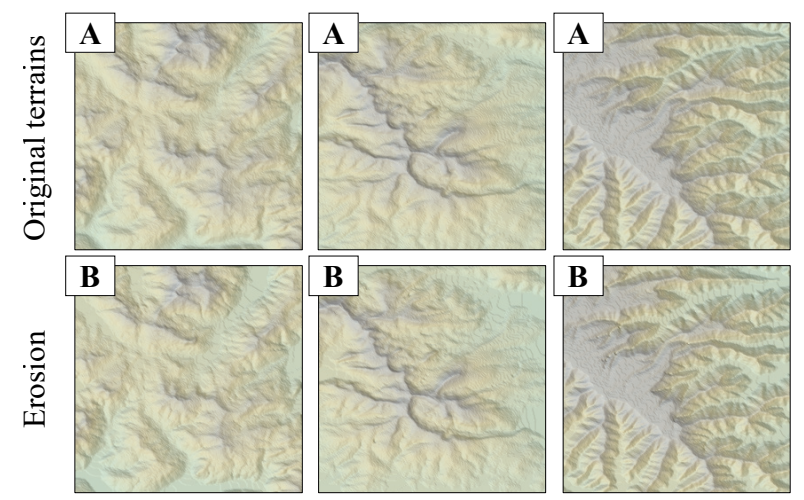

Fig. 12. Erosion synthesizer training pair examples.

Although it may appear counter-intuitive to learn a process that can be simulated, our goal is to take advantage of the efficiency of synthesizers at run time to provide interactive feedback to the user. This method is an approximation of complex erosion phenomena that runs extremely fast as opposed to computationally demanding simulations. The idea of simulating complex and hard-to-simulate phenomena using neural network is inspired by learning computationally expensive iterative processes such as image filters [Xu et al. 2015] and style transfer [Johnson et al. 2016; Ulyanov et al. 2016].

\section{AUTHORING}

Interactive authoring takes place after the network training preprocessing step and it is a two-step workflow (Fig. 3). The user first draws a coarse sketch and incrementally edits it by adding, modifying, and removing curves or carving level-sets. The user then refines the terrain by using optional erosion and amplification that generates the final high resolution model.

\subsection{Terrain Sketching}

Our system uses the terrain synthesizers to generate terrains corresponding to the user-provided sketch at runtime. The user initially sketches a river network, a ridge network, elevation cues, or any combinations of the three and the synthesizer generates a terrain. The user may add or modify ridges, rivers, elevation cues or remove some parts of the sketch and see the results in real time as applying 

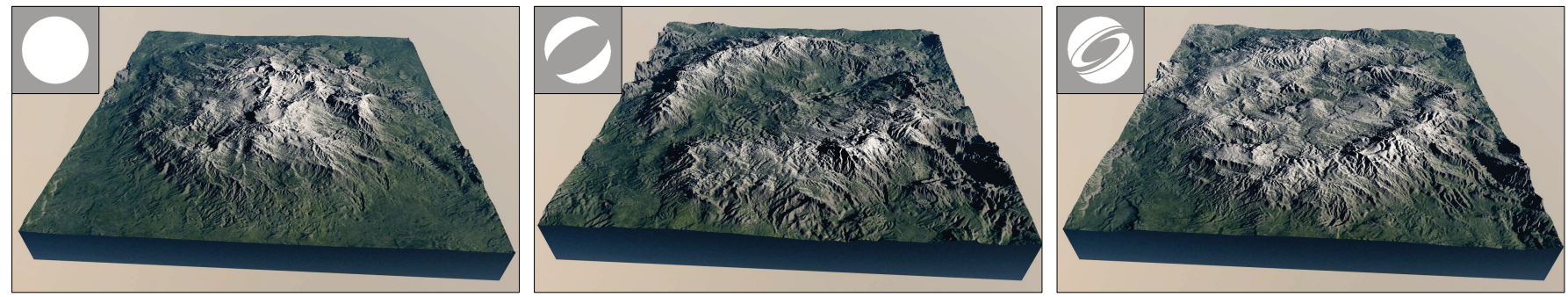

Fig. 13. The iterative sketching can be used to generate complex shapes. Here the user sketches the Siggraph logo by adding a disk, carving a part of the levelset out, and finally adding details. This whole editing sequence is performed using the levelset-to-terrain synthesizer

the terrain synthesizer is very efficient and takes around $50 \mathrm{~ms}$ per generation.

An example in Fig. 10 shows an authoring session of an artist providing initial ridges, then adding rivers, and finally elevation cues. Another example in Fig. 2 shows an authoring session by a novice user. The user first defined ridges, then added rivers, and modified the terrain by providing a set of altitude cues.

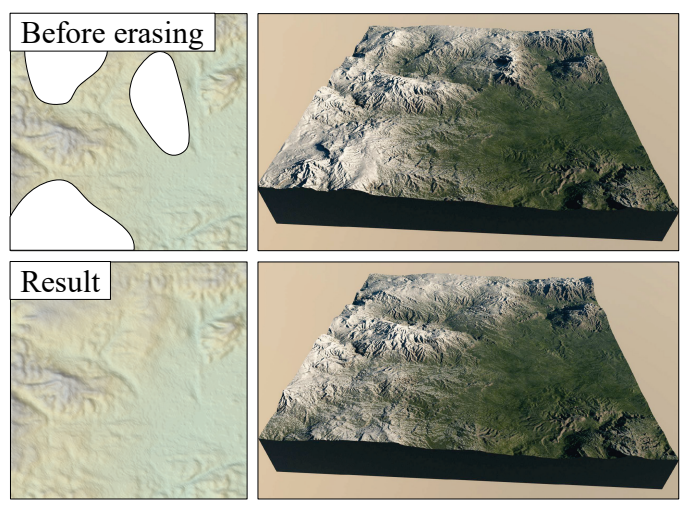

Fig. 14. Example of a terrain automatically generated by the eraser synthesizer tool that fills parts removed by the user.

A key feature of our approach is that it allows the user to use different types of sketching models as input. Instead of sketching with ridge and river curves, the user can interactively edit a levelset, and use the corresponding synthesizer to generate the terrain. Fig. 13 shows three consecutive steps of an interactive level set authoring session: starting from a circular shape, the user erased the center before adding more details. This drawing tool is fast and easy to use, however it provides less control than the curve sketches. The eraser synthesizer can quickly regenerate terrains with missing parts and produces consistent models (Fig. 14).

\subsection{Terrain Refinement}

Terrain refinement encompasses the processes of erosion and amplification that improve the overall realism of the generated terrain and increase its resolution.
Erosion: Once the coarse sketch terrain is provided, the user can apply erosion to it. The erosion synthesizer mimics erosion algorithms at a very small computational cost as opposed to numerical simulations. Fig. 15 illustrates the simulated erosion on real world terrains and compares it with the learned erosion. The computation time of our trained erosion-synthesizer is three order of magnitude faster $(25 \mathrm{~ms}$ vs $40,000 \mathrm{~ms}$ on a terrain of resolution $256 \times 256)$ compared to a simulated erosion.
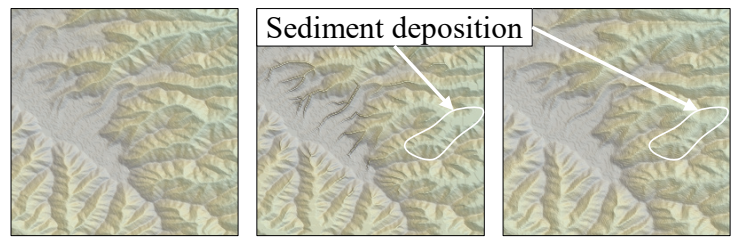

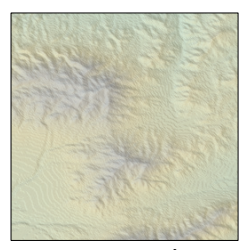

Input terrains

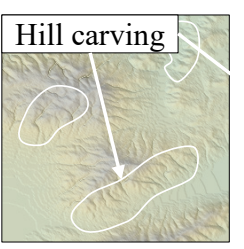

Erosion simulation

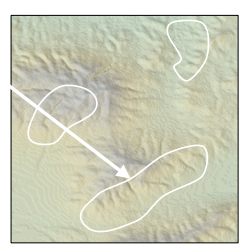

Inferred terrains
Fig. 15. Simulating the erosion of a terrain comes at a very small cost at runtime.

Terrain amplification: After the large-scale terrain has been generated and erosion has possibly been applied, the final step is to add more details by using terrain amplification (see Section 3). We use the patch-based amplification method proposed in [Guérin et al. 2016] that builds high and low resolution patch dictionaries and decomposes the terrain onto them. Although it would be possible to also train a terrain synthesizer for the amplification, this would require learning several synthesizers for each resolution gain, whereas the sparse-amplification performs this operation very efficiently. Because the cGANs generate coherent large-scale terrains, the terrain amplification is well-suited to match terrain details to large scale terrain patches (Fig. 16).

\subsection{Integration with Large Scale Terrain Modeling}

A key feature of our approach is its ability to analyze input DEMs and generate sketch representations which can be used as input data 


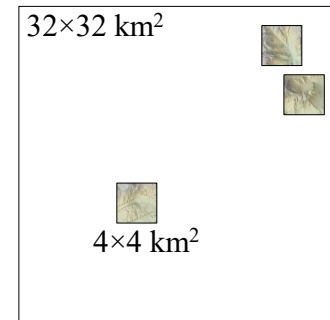

Initial setup

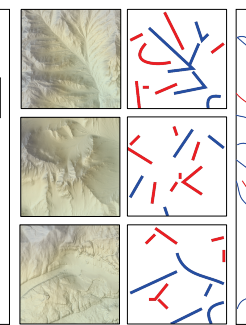

Patch analysis

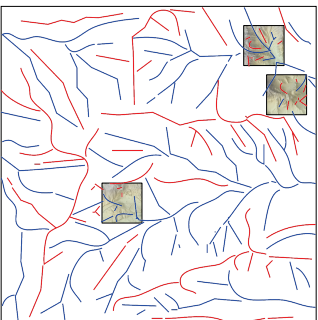

Sketching

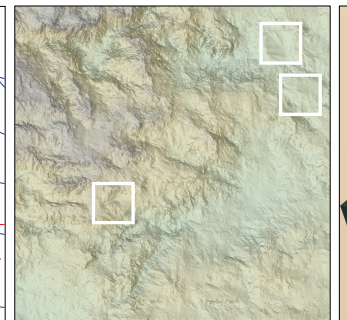

Generated terrain

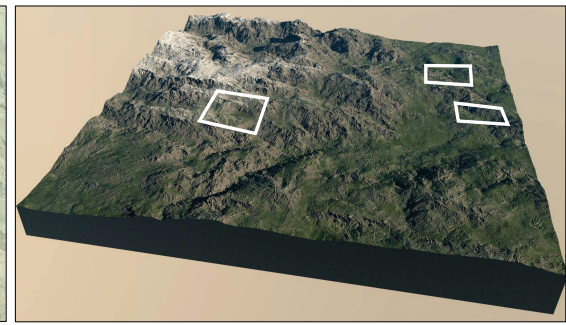

Final rendering

Fig. 17. Our method can generate terrains from sketches that have vast empty areas. In the initial setup, three small terrain patches of $4 \times 4 \mathrm{~km}^{2}$ were carefully authored and located by the designer on the large empty square terrain of size $32 \times 32 \mathrm{~km}^{2}$. The analysis of the patches produced the initial local set of local ridges, rivers, and elevation landmarks, which were completed by user-defined sketches over the remainder of the domain. The terrain was automatically generated by the sketch-synthesizer $\mathcal{S}$, and the patches blended with the terrain.

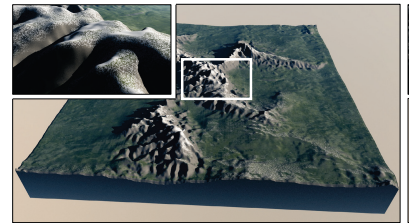

Synthesizer output

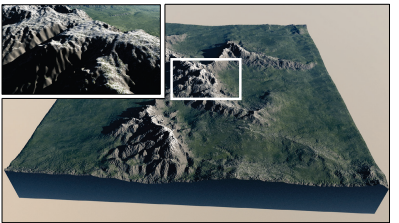

$4 \times$ amplified terrain
Fig. 16. Comparison between a synthesized terrain before (left) and after amplification (right). Terrain amplification process introduces details without breaking the landform features generated by the synthesizer as can be seen in the image inset.

in the synthesizers $\mathcal{S}$. Our method enables artists to generate large scale terrains featuring some specific regions that they authored in full detail in a seamless fashion. The overall process proceeds in three steps (Fig. 17). First, given several high-resolution input DEM patches $\mathbf{A}_{k}$ embedded in a larger domain $\Omega$, we down-sample and analyze them to produce their corresponding low-resolution curve or levelset sketches $\mathbf{B}_{k}$. The user then completes the coarse sketch over the remainder of the domain $\Omega-\left(\cup_{k} \mathbf{A}_{k}\right)$ to get a new representation $\widetilde{\mathbf{B}}$. Finally, we generate the terrain $\mathbf{A}$ from $\widetilde{\mathbf{B}}$ and locally smoothly blend it with the prescribed patches $\mathbf{A}_{k}$.

The analysis of the features of the detailed input patches allows us to obtain a large scale DEM whose major landform features are consistent with the prescribed patches' ones. Therefore, the final blending, although simple and straightforward, generates a coherent DEM. Fig. 17 shows an example of our high level and very efficient authoring approach. The professional artist created the $32 \times 32 \mathrm{~km}^{2}$ terrain featuring three specific landforms in less than 15 minutes.

\section{RESULTS AND DISCUSSION}

Generation of the training database has been implemented in Matlab ${ }^{\circledR}$ and $\mathrm{C}++$ and interfaced with TensorFlow for the Deep Learning part. We adapted the cGAN code provided by the authors of [Isola et al. $2016]$ to process DEM data. Training was performed on a NVidia ${ }^{\circledR}$ Titan X graphics card with $12 \mathrm{~Gb}$ of memory clocked at $1.076 \mathrm{Ghz}$. Our interactive editing application was implemented in $\mathrm{C}++$ and uses Qt and OpenGL for rapid previsualization. Interactive editing performed on a standard desktop computer equipped with an Intel Core i7 CPU clocked at $3.4 \mathrm{GHz}$ and with a NVidia ${ }^{\circledR}$ GTX 970. For visualization purposes, we added a procedural texture to our synthesized terrains. The photorealistic landscape images were rendered with the $V u e^{\circledR}$ software. Unless stated otherwise, all high quality results use amplification.

\subsection{Database and training}

Our real-world terrain database includes DEMs extracted from USGS Earth explorer. We used 35 patches of one square degree at a precision of one arc-second taken from NASA SRTM. Each patch consists of a 3, $600 \times 3,600$ resolution grid and each cell represents horizontal area of approximately $30 \times 30$ meters. We used 16 bits gray-scale in our implementation with a vertical resolution of $1 \mathrm{~m}$. Table 1 reports statistics for generating the different databases and training the network.

\begin{tabular}{|c|c|c|c|}
\hline \multirow{2}{*}{ Synthesizer } & \multicolumn{2}{|c|}{ Database creation } & \multirow{2}{*}{$\begin{array}{c}\text { Training } \\
\text { time }\end{array}$} \\
\hline & Size & Time & \\
\hline Sketch-to-Terrain & 525 & $0: 22$ & $6: 25$ \\
\hline Levelset-to-Terrain & 525 & 0:01 & $6: 24$ \\
\hline Eraser & 500 & 0:01 & $5: 48$ \\
\hline Erosion & 1400 & $15: 13$ & $6: 54$ \\
\hline
\end{tabular}

Table 1. Timings (in hours) for the learning of terrain synthesizers.

The structure of the generator network is linked to the input terrain resolution, therefore all generated terrains will have the same resolution. Because of the fully-convolutional nature of the synthesizer, we are able to generate terrains from sketches of arbitrary size. While the spatial resolution will remain unchanged (i.e., the pixel size will represent the same distance), the total size of the synthesized terrain can be larger. The only limitation of this process is the amount of GPU memory. In our implementation, we were able to synthesize terrains up to a resolution of $1024 \times 1024$. 


\subsection{Comparisons}

In this section, we compare our method to other sketching tools, and evaluate the performance of our erosion synthesizer with respect to physically-based simulations

Several terrain sketching algorithms have been proposed in computer graphics. Zhou et al. [2007] proposed an example-based terrain authoring method based on texture synthesis techniques that generates terrains by combining patches from an input sketch and mountain range style image. Here we reproduce the lambda-shaped mountain range from their work that was sketched as a ridge without any additional information (Fig. 18). We used the sketch-toterrain synthesizer on the sketch to generate the final terrain. It is important to note that our cGAN-based method allows for an interactive editing and does not require any input patches from the terrain. The features obtained by [Zhou et al. 2007] are slightly sharper than ours. We obtain similar small-scale sharp ridges and faults when completing our deep-learning-based terrain generation with amplification.

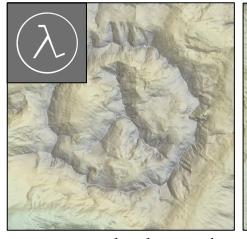

Example-based

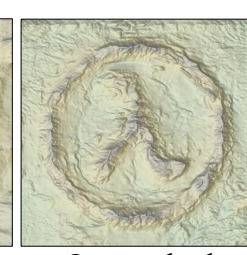

Our method
Fig. 18. Comparison between the example-based method of [Zhou et al. 2007] and our method.

Hnaidi et al. [2010] proposed a terrain sketching approach that also uses sketches of ridges and rivers networks to synthesize a terrain using diffusion. An important limitation of this method lies in the amount of information that is required to generate the terrain: rivers and ridges should be precisely sketched and their elevations should be prescribed (Fig. 19). In contrast, our approach generates realistic terrains even from coarse sketches, because the synthesizers learned to generate more details from less input information. Without any derivative constraints on the ridges, Hnaidi et al.[2010] amounts to a simple heat diffusion yielding unrealistic smooth plateaus (Fig. 19).
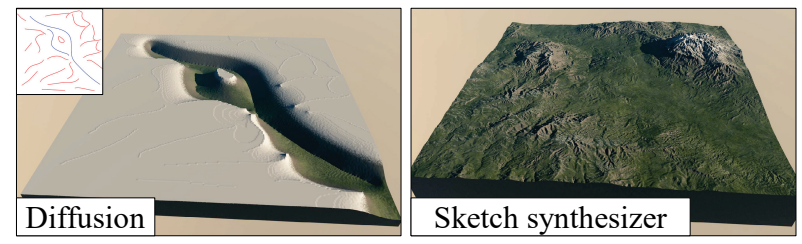

Fig. 19. Comparison between the diffusion method of [Hnaidi et al. 2010] (left) and our sketch synthesizer (right). Without additional gradient information and noise parameters, the diffusion-based method produces unnatural smooth terrains.
We further compare with the work [Guérin et al. 2016] (Fig. 20). The input is a levelset binary image that is fed to the leveset-toterrain synthesizer for our method, while it is first smoothed then processed by the sparse terrain approach since this method requires a smooth sketch as input. Our approach manages to synthesize plausible terrains even at large scales, whereas the sparse modeling approach yields an unrealistic result because it does not take into account large scale features.

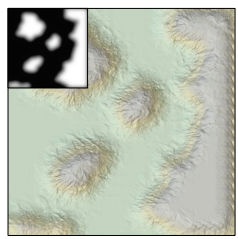

Sparse method

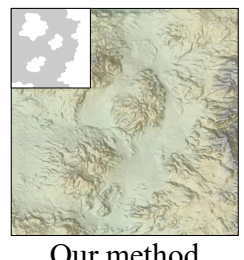

Our method
Fig. 20. Comparison between the sparse method from [Guérin et al. 2016] and our levelset synthesizer. Our method produces a vast variety of landforms features whereas sparse modeling reproduces similar details.

We also compared our results with baseline patch-based method based on the PatchMatch algorithm [Barnes et al. 2009] that finds approximate image patch correspondences. The input was first sketched by using heat diffusion and then matched to an $901 \times 901$ exemplar terrain; a large terrain tile used in our dataset. To allow for better correspondences, the terrain exemplar was blurred for the matching step and used in its full resolution for the reconstruction step. Two synthesis examples are shown in Fig. 21. The first is the traditional reconstruction minimizing the bidirectional similarity (BDS) metric defined in [Barnes et al. 2009] by copying pixel values from the terrain exemplar. The second one matches gradient values instead of pixel values followed by BDS minimization and Poisson solving. While direct PatchMatch reconstruction exhibits strong patch artifacts, the gradient-based reconstruction generates fewer artifacts, but fails at recovering smaller-scale structures provided by the exemplar terrain. Our method quickly shows large and medium-scale features but fails at generating small-scale features.

To show the efficiency of our example-based approach, we also compare the results of our trained erosion synthesizer with erosion simulation. Our algorithm generates a terrain that looks eroded, but

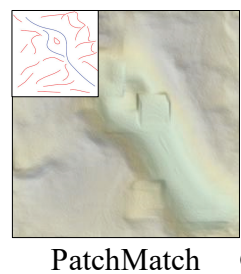

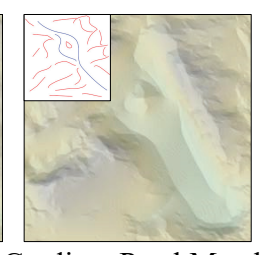

Gradient PatchMatch

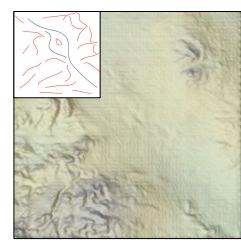

Our method
Fig. 21. Comparisons with baseline patch-based methods: left image shows the direct reconstruction (10s), middle shows gradient-based reconstruction (15s), right shows the terrain generated by our method (25ms). Our method quickly shows large and medium scale features but needs further processing to generate small-scale features. 


\begin{tabular}{|l|c|r|}
\hline Process & Terrain Size & Time $(\mathrm{ms})$ \\
\hline & $256 \times 256$ & 25 \\
Synthesizers $\mathcal{L}$ and $\mathcal{S}$ & $512 \times 512$ & 55 \\
& $1024 \times 1024$ & 190 \\
\hline Optional erosion $\mathcal{E}$ or eraser $\mathcal{R}$ & $1024 \times 1024$ & 190 \\
\hline Interactive feedback & $512 \times 512$ & 310 \\
\hline Optional amplification $(\times 4)$ & $256^{2} \rightarrow 1024^{2}$ & 800 \\
& $512^{2} \rightarrow 2048^{2}$ & 3250 \\
\hline
\end{tabular}

Table 2. Statistics for interactive authoring: terrain size and processing time (in ms). The levelset $\mathcal{L}$, curve sketch $\mathcal{S}$, erosion $\mathcal{E}$ and eraser $\mathcal{R}$ synthesizers perform in a few milliseconds and allow for interactive editing. The whole interactive feedback includes all the processes that are necessary to obtain the final shaded terrain in the interface after a user stroke. Amplification slows down the overall process and is usually disabled during interactive editing.

it may contain some geologically incorrect features as compared to the real erosion phenomenon. However this approximation is sufficient for many applications that only need plausible terrains (Fig. 22). Our implementation of the cGAN-based method performs $4,000 \times$ faster than erosion simulations.
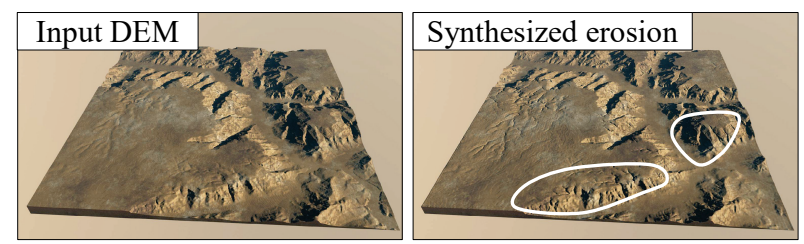

Fig. 22. Large scale terrain erosion (left) and erosion synthesizer output (right) on a $1024 \times 1024$ terrain. Erosion simulation took $741.0 \mathrm{~s}$ whereas our method took $0.7 \mathrm{~s}$.

\subsection{Performance, User Control, and Experience}

Our approach provides the user with an intuitive and simple control and allows inexperienced users to create plausible terrains with a few strokes as illustrated in Fig. 23. The speed of the generation process allows for interactive modeling and the sketches can be quickly modified by adding and removing features or by using the eraser (see Table 2). Moreover, amplification can be enabled or disabled independently of the sketching process. The conformity between the user sketch and the generated terrain is high as illustrated in Fig. 23, where a real-world terrain is reproduced by iterative sketching. Although the overall dynamic of the terrain is easy to reproduce, it takes many more strokes to get closer to the target.

Novice and expert artists tested our interactive system. We observed that the usability was simple because all participants were able to express their intent after only a few $(2-3)$ short interactive sessions. All users (novice and expert) were particularly satisfied with the simplicity of the interface (see the accompanying video), the interactive feedback, and the variety of terrain models they could design (Fig. 24, 27). The ridges, rivers, and altitude cues sketching
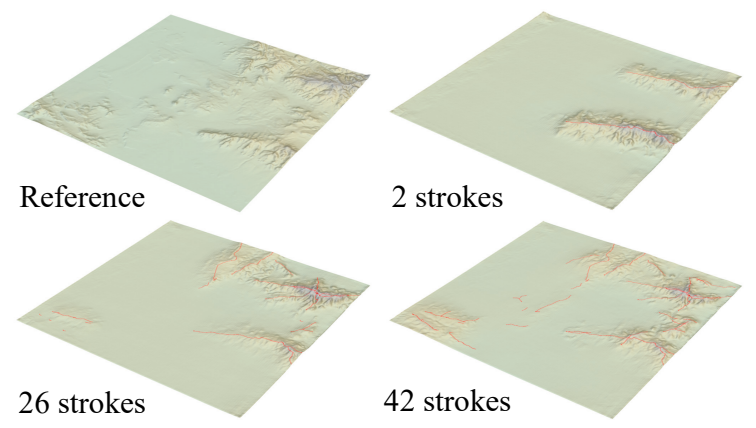

Fig. 23. Reproducing a real terrain. Although the overall dynamic of the terrain can be captured in a few strokes, it takes a denser sketch to reproduce both large-scale and medium-scale features of the terrain.

tools were considered helpful and satisfactory to the requirements expected by expert artists.

Moreover, we conducted a qualitative user-study with five users. We first explained the interface and the users were invited to practice during a few minutes. Then we asked the users to draw three different scenes described textually as follows: a centered high mountain with small mountains around it, a mountain range traversing the scene diagonally, and a volcano. At the end of the experiment, the participants were asked to evaluate the system on a 1 to 4 Likert scale according to three criteria: 1) Does the generated terrain follow the sketch? 2) Is the system reactive? 3) Is it easy to express ones intent? We also let the users express their remarks about the system.

On the criteria 1) and 2), all the participants answered 1 or 2 (strong agree and agree). On the criterion 3), the participants answered also 1 or 2 except for one who answered 3 (disagree). Some users pointed out that they would have liked an additional tool to produce smoother and more regular slopes. They also noticed that using multiple strokes was useful to strengthen a ridge, but had a side effect of lowering the influence of the other strokes.

\subsection{Limitations and Failure Cases}

The main limitation of our method is that each synthesizer is dedicated to a single task. If one wants to use a different kind of sketch, a new synthesizer should be added and trained. Thus, the user must learn to draw a certain type of sketch that is captured by the synthesizers. However, as stated above, the sketch adaptation is intuitive and was quickly understood by the users who were able to obtain satisfactory terrains matching their ideas. Future work could target online adaptation of terrain synthesizers to users, and let them specify definitions of sketch elements.

There are several situations when our algorithm fails to produce realistic results. First, if the sketch is sparse, a strong repetition effect will appear as shown in Fig. 25. This artifact can be alleviated by adding more strokes. Second, in planar areas or when no sketch cues is available the synthesized terrain may exhibit some regular pattern. This is an artifact of the cGAN training, which can be alleviated in a post-processing step by applying a simple $5 \times 5$ median filter as shown in Fig. 26. This can be further improved by our terrain amplification. Finally, a limitation of our interface design 

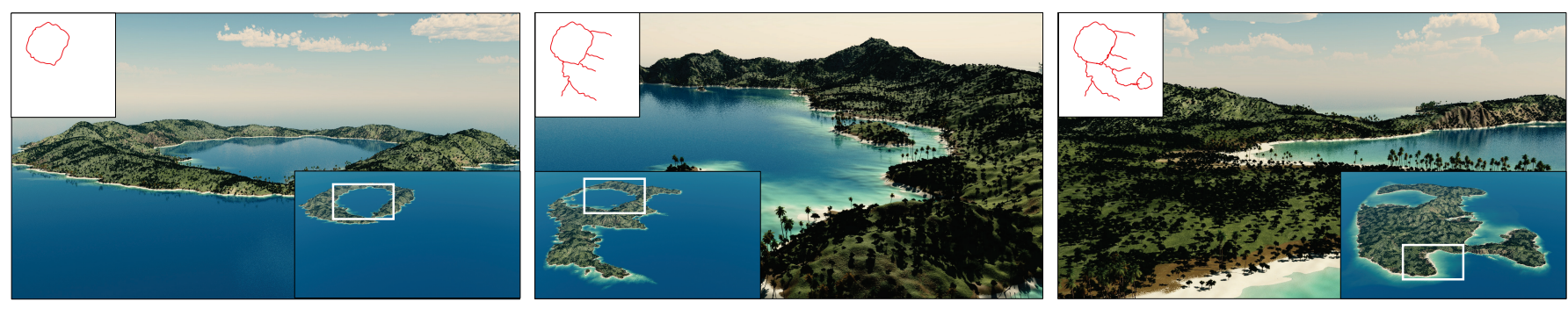

Fig. 24. An island authored in a few minutes with only 11 strokes by a novice user. Notice how the long sketched ridge is well preserved by our method.

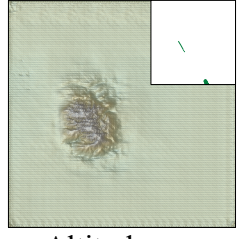

Altitude cue

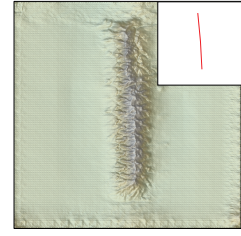

Ridge

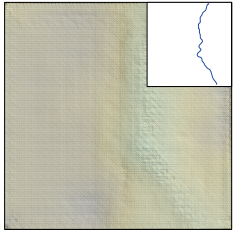

River
Fig. 25. Failure case: sometimes when the input sketch is too sparse, our method generates repeated terrain patches and grid artifacts.

is that levelset and curve sketches cannot be used simultaneously. Combining both would make the editing more demanding, since the levelset structure should be coherent with the altitude cues, the ridges, and the river network.

\section{CONCLUSION}

We introduced a novel framework for modeling terrains from input sketches. Our approach enables users to create large scale realistic models quickly and easily, without the need of writing procedural rules or defining the parameters of physically-based simulations. Given a large set of terrain examples, we automatically extract ridge and river network curves, eroded terrain models and other characteristics data that are used for training cGAN networks. During the interactive authoring session, the user sketches important features corresponding to his intent, and the cGAN generates a realistic terrain. This process is very efficient: each terrain generation takes only a few milliseconds which allows interactive feedback to the designer. Our approach merges procedural modeling and interactive sketching in a unified framework, bridging the gap between the intuitiveness and flexibility of interactive authoring processes, while using the expressive power of examples that can be either real-world or procedural.

At the heart of our method lies the possibility of learning correspondences between the characteristic features of a terrain and its elevation data. An interesting extension of our work would be to bind a procedural model to our system, such as the procedural primitive-based terrain representation proposed in [Génevaux et al. 2015] and learn the parameters so as to obtain a complete inverse procedural modeling system. Another promising future work is to generalize our approach to model terrains with different material layers such as bedrock, rock, sand or humus, and vegetation.

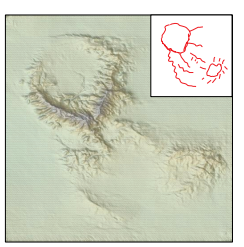

Synthesizer output

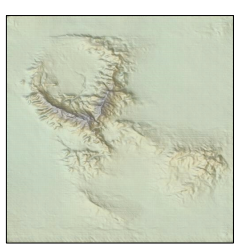

Median filtering

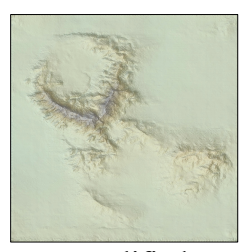

Amplified
Fig. 26. Failure case: a regular grid pattern appears when the terrain is flat due to a lack of input cues. This can be alleviated by using a median filter: the pattern is removed and after amplification no trace of it can be seen.

\section{REFERENCES}

Connelly Barnes, Eli Shechtman, Adam Finkelstein, and Dan B. Goldman. 2009. PatchMatch: A Randomized Correspondence Algorithm for Structural Image Editing. ACM Transactions on Graphics (Proc. SIGGRAPH) 28, 3 (Aug. 2009).

Farès Belhadj and Pierre Audibert. 2005. Modeling landscapes with ridges and rivers: bottom up approach. In GRAPHITE. 447-450.

Bedrich Benes and Rafael Forsbach. 2001. Layered Data Representation for Visual Simulation of Terrain Erosion. In Proceedings of the 17th Spring conference on Computer graphics. 80-85.

Bedrich Benes, Václav Těšínský, Jan Hornyš, and Sanjiv K. Bhatia. 2006. Hydraulic erosion. Comput. Animat. Virtual Worlds 17, 2 (2006), 99-108.

Norishige Chiba, Kazunobu Muraoka, and Kunihiko Fujita. 1998. An erosion model based on velocity fields for the visual simulation of mountain scenery. Fournal of Visualization and Computer Animation 9, 4 (1998), 185-194.

Guillaume Cordonnier, Jean Braun, Marie-Paule Cani, Bedrich Benes, Éric Galin, Adrien Peytavie, and Éric Guérin. 2016. Large Scale Terrain Generation from Tectonic Uplift and Fluvial Erosion. Computer Graphics Forum 35, 2 (2016), 165-175.

Guillaume Cordonnier, Marie-Paule Cani, Bedrich Benes, Jean Braun, and Eric Galin. 2017a. Sculpting Mountains: Interactive Terrain Modeling Based on Subsurface Geology. IEEE Transactions on Visualization and Computer Graphics PP, 99 (2017), $1-1$.

Guillaume Cordonnier, Eric Galin, James Gain, Bedrich Benes, Eric Guérin, Adrien Peytavie, and Marie-Paule Cani. 2017b. Authoring Landscapes by Combining Ecosystem and Terrain Erosion Simulation. ACM Transactions on Graphics 36, 4 (2017).

Evgenij Derzapf, Björn Ganster, Michael Guthe, and Reinhard Klein. 2011. River Networks for Instant Procedural Planets. Computer Graphics Forum 30, 7 (2011), 2031-2040.

Alexey Dosovitskiy, Jost Tobias Springenberg, and Thomas Brox. 2015. Learning to Generate Chairs with Convolutional Neural Networks. In CVPR.

Alain Fournier, Don Fussell, and Loren Carpenter. 1982. Computer rendering of stochastic models. Commun. ACM 25, 6 (1982), 371-384.

James Gain, Patrick Marais, and Wolfgang Strasser. 2009. Terrain sketching. In Proc. Symposium on Interactive 3D Graphics and Games - I3D. ACM, 31-38.

James E. Gain, Bruce Merry, and Patrick Marais. 2015. Parallel, Realistic and Controllable Terrain Synthesis. Computer Graphics Forum 34, 2 (2015), 105-116.

Leon A. Gatys, Alexander S. Ecker, and Matthias Bethge. 2015. Texture synthesis and the controlled generation of natural stimuli using convolutional neural networks. CoRR abs/1505.07376 (2015)

Jean-David Génevaux, Eric Galin, Eric Guérin, Adrien Peytavie, and Bedrich Benes. 2013. Terrain Generation Using Procedural Models Based on Hydrology. ACM Transaction on Graphics 32, 4 (2013), 143:1-143:13. 

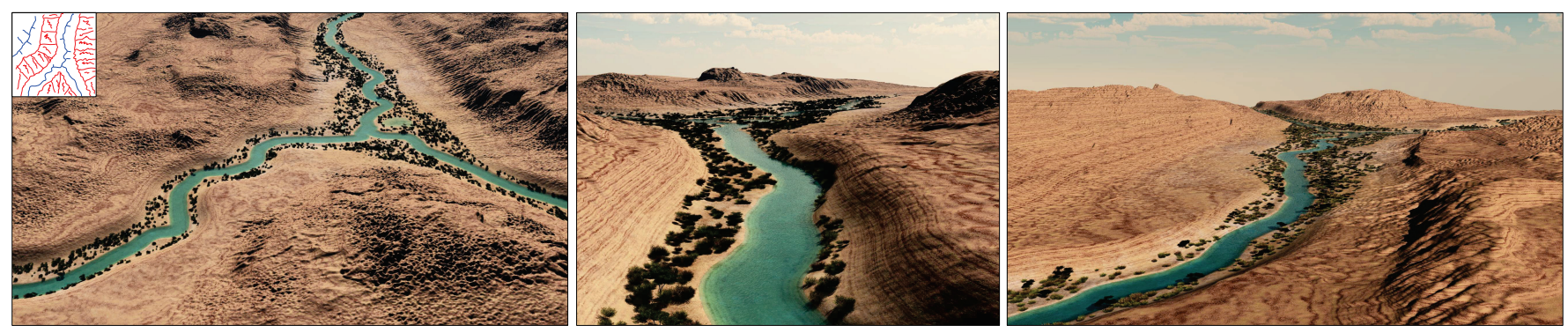

Fig. 27. The canyon was authored by a novice user in less than two minutes. The riverbed was carved using river primitives [Génevaux et al. 2015] created from the user-defined input curve.

Jean-David Génevaux, Eric Galin, Adrien Peytavie, Eric Guérin, Cyril Briquet, François Grosbellet, and Bedrich Benes. 2015. Terrain Modelling from Feature Primitives. Computer Graphics Forum 34, 6 (2015), 198-210.

Ian J. Goodfellow, Jean Pouget-Abadie, Mehdi Mirza, Bing Xu, David Warde-Farley, Sherjil Ozair, Aaron C. Courville, and Yoshua Bengio. 2014. Generative Adversarial Nets. In Proc. Annual Conference on Neural Information Processing Systems (NIPS) 2014. 2672-2680.

Karol Gregor, Ivo Danihelka, Alex Graves, and Daan Wierstra. 2015. DRAW: A Recurrent Neural Network For Image Generation. In ICML.

Eric Guérin, Julie Digne, Eric Galin, and Adrien Peytavie. 2016. Sparse representation of terrains for procedural modeling. Computer Graphics Forum (Proceedings of Eurographics) 35, 2 (2016), 177-187.

Houssam Hnaidi, Eric Guérin, Samir Akkouche, Adrien Peytavie, and Eric Galin. 2010 Feature based terrain generation using diffusion equation. Computer Graphics Forum (Proceedings of Pacific Graphics) 29, 7 (2010), 2179-2186.

Haibin Huang, Evangelos Kalogerakis, Ersin Yumer, and Radomír Mech. 2016. Shape Synthesis from Sketches via Procedural Models and Convolutional Networks. IEEE Transactions on Visualization and Computer Graphics PP, 99 (2016), 1-1.

Sergey Ioffe and Christian Szegedy. 2015. Batch Normalization: Accelerating Deep Network Training by Reducing Internal Covariate Shift. ICML.

Phillip Isola, Jun-Yan Zhu, Tinghui Zhou, and Alexei A. Efros. 2016. Image-to-Image Translation with Conditional Adversarial Networks. arxiv (2016).

Justin Johnson, Alexandre Alahi, and Li Fei-Fei. 2016. Perceptual Losses for Real-Time Style Transfer and Super-Resolution. Springer, Cham, 694-711.

Alex Kelley, Michael Malin, and Gregory Nielson. 1988. Terrain simulation using a model of stream erosion. In Proceedings of SIGGRAPH. 263-268.

Diederik P. Kingma and Jimmy L. Ba. 2015. Adam: A Method for Stochastic Optimization ICLR.

Diederik P Kingma and Max Welling. 2014. Auto-encoding variational bayes. In ICLR.

Peter Krištof, Bedrich Benes, Jaroslav Křivánek, and Ondřej Štavava. 2009. Hydraulic Erosion Using Smoothed Particle Hydrodynamics. Computer Graphics Forum (Proceedings of Eurographics) 28, 2 (2009), 219-228.

Alex Krizhevsky, Ilya Sutskever, and Geoffrey E. Hinton. 2012. ImageNet Classification with Deep Convolutional Neural Networks. In Advances in Neural Information Processing Systems 25. Curran Associates, Inc., 1097-1105.

Vivek Kwatra, Arno Schödl, Irfan Essa, Greg Turk, and Aaron Bobick. 2003. Graphcut Textures: Image and Video Synthesis Using Graph Cuts. ACM Transactions on Graphics, SIGGRAPH 2003 22, 3 (2003), 277-286.

Chuan Li and Michael Wand. 2016. Precomputed Real-Time Texture Synthesis with Markovian Generative Adversarial Networks. Springer International Publishing, Cham, 702-716.

Elman Mansimov, Emilio Parisotto, Jimmy L. Ba, and Ruslan Salakhutdinov. 2016 Generating Images from Captions with Attention. In ICLR.

Michaël Mathieu, Camille Couprie, and Yann LeCun. 2016. Deep multi-scale video prediction beyond mean square error. In ICLR.

Xing Mei, Philippe Decaudin, and Baogang Hu. 2007. Fast Hydraulic Erosion Simulation and Visualization on GPU. In Pacific Graphics. IEEE, 47-56.

Mehdi Mirza and Simon Osindero. 2014. Conditional Generative Adversarial Nets. CoRR abs/1411.1784 (2014).

Forest K. Musgrave, Craig E. Kolb, and Robert S. Mace. 1989. The synthesis and rendering of eroded fractal terrains. In Proceedings of SIGGRAPH. 41-50.

Kenji Nagashima. 1998. Computer generation of eroded valley and mountain terrains. The Visual Computer 13, 9-10 (1998), 456-464.

Mattia Natali, Endre M. Lidal, Julius Parulek, Ivan Viola, and Daniel Patel. 2013. Modeling Terrains and Subsurface Geology. In EuroGraphics 2013 State of the Art Reports (STARs). 155-173.
Gen Nishida, Ignacio Garcia-Dorado, Daniel G. Aliaga, Bedrich Benes, and Adrien Bousseau. 2016. Interactive Sketching of Urban Procedural Models. ACM Trans. Graph. 35, 4 (2016), 130:1-130:11.

John O'Callaghan and David Mark. 1984. The extraction of drainage networks from digital elevation data. Comput. Vis. Graph. Image Process 28, 3 (1984), 323-344.

Deepak Pathak, Philipp Krähenbühl, Jeff Donahue, Trevor Darrell, and Alexei Efros. 2016. Context Encoders: Feature Learning by Inpainting. In CVPR.

Adrien Peytavie, Eric Galin, Stephane Merillou, and Jerome Grosjean. 2009. Arches: a Framework for Modeling Complex Terrains. Computer Graphics Forum (Proceedings of Eurographics) 28, 2 (2009), 457-467.

Przemyslaw Prusinkiewicz and Marc Hammel. 1993. A fractal model of mountains with rivers. In Graphics Interface. 174-180.

Alec Radford, Luke Metz, and Soumith Chintala. 2016. Unsupervised Representation Learning with Deep Convolutional Generative Adversarial Networks.

Olaf Ronneberger, Philipp Fischer, and Thomas Brox. 2015. U-Net: Convolutional Networks for Biomedical Image Segmentation. Springer International Publishing, 234-241.

Ron Rubinstein, Alfred M. Bruckstein, and Michael Elad. 2010. Dictionaries for Sparse Representation Modeling. Proc. IEEE 98, 6 (June 2010), 1045-1057.

Ruben M. Smelik, Tim Tutenel, Rafael Bidarra, and Bedrich Benes. 2014. A Survey on Procedural Modelling for Virtual Worlds. Computer Graphics Forum 33, 6 (2014), 31-50.

David G. Tarboton, Rafael L. Bras, and Ignacio Rodriguez-Iturbe. 1991. On Extraction of Channel Networks From Digital Elevation Data. Hydrological Processes 5, 3 (1991), 81-100.

Flora Ponjou Tasse, Arnaud Emilien, Marie-Paule Cani, Stefanie Hahmann, and Adrien Bernhardt. 2014. First Person Sketch-based Terrain Editing. In Proceedings of Graphics Interface. 217-224.

Flora Ponjou Tasse, James Gain, and Patrick Marais. 2012. Enhanced Texture-Based Terrain Synthesis on Graphics Hardware. Computer Graphics Forum 31, 6 (2012), 1959-1972.

Dmitry Ulyanov, Vadim Lebedev, Andrea Vedaldi, and Victor S. Lempitsky. 2016. Texture Networks: Feed-forward Synthesis of Textures and Stylized Images. (2016).

Juraj Vanek, Bedrich Benes, Adam Herout, and Ondřej Štava. 2011. Large-Scale PhysicsBased Terrain Editing Using Adaptive Tiles on the GPU. Computer Graphics and Applications 31, 6 (2011), 35-44.

Ondřej Štava, Bedrich Benes, Matthew Brisbin, and Jaroslav Křivánek. 2008. Interactive Terrain Modeling Using Hydraulic Erosion. In ACM Siggraph/Eurographics Symposium on Computer Animation. 201-210.

Li Xu, Jimmy Ren, Qiong Yan, Renjie Liao, and Jiaya Jia. 2015. Deep Edge-Aware Filters. In Proceedings of the 32nd International Conference on Machine Learning (Proceedings of Machine Learning Research), Francis Bach and David Blei (Eds.), Vol. 37. PMLR, 1669-1678.

Howard Zhou, Jie Sun, Greg Turk, and James M. Rehg. 2007. Terrain Synthesis from Digital Elevation Models. IEEE Transactions on Visualization and Computer Graphics 13, 4 (2007), 834-848.

Jun-Yan Zhu, Philipp Krähenbühl, Eli Shechtman, and Alexei A. Efros. 2016. Generative Visual Manipulation on the Natural Image Manifold. In Proceedings of European Conference on Computer Vision (ECCV). 